\title{
On Choquet Integrals with Respect to a Fuzzy Complex Valued Fuzzy Measure of Fuzzy Complex Valued Functions
}

\author{
Lee-Chae Jang* and Hyun-Mee Kim ${ }^{* * *}$ \\ "Dept. of Mathematics and Computer Science, Konkuk University \\ Chungju 380-701, Korea, E-mail:leechae.jang@kku.ac.kr \\ "Dept. of Mathematics, Kyunghee University \\ Seoul 130-701, Korea, E-mail: kagness@khu.ac.kr
}

\begin{abstract}
In this paper, using fuzzy complex valued functions and fuzzy complex valued fuzzy measures ([11]) and interval-valued Choquet integrals ([2-6]), we define Choquet integral with respect to a fuzzy complex valued fuzzy measure of a fuzzy complex valued function and investigate some basic properties of them.
\end{abstract}

Key Words : fuzzy numbers, comonotonic, fuzzy complex numbers, fuzzy complex valued function, fuzzy complex valued fuzzy measures, Choquet integrals.

\section{Introduction}

Buckley [1] first defined the concept of fuzzy complex numbers and have studied the theory of fuzzy complex numbers, the differentiability and integrability of fuzzy complex valued functions on a complex plane $\mathbb{C}$. Wang and $\mathrm{Li}$ [11] studied generalized Lebesgue integrals of fuzzy complex valued functions.

By using the method of establishing the basic framework for fuzzy complex analysis, we will define Choquet integrals with respect to a fuzzy complex valued fuzzy measure of fuzzy complex valued functions. We note that interval-valued Choquet inetgrals were defined by Jang [2-6].

Let $(X, \Omega)$ be a measurable space. A mapping $\mu: \Omega \rightarrow[0, \infty]$ on $X$ is called a fuzzy measure if it is satisfying the following conditions;

(i) $\mu(\varnothing)=0$,

(ii) $\mu(A) \leq \mu(B)$,

whenever $A, B \in \Omega, A \subset B$.

(iii) for every increasing sequence $\left\{A_{n}\right\}$ of measurable sets, we have

$$
\mu\left(\cup_{n=1}^{\infty} A_{n}\right)=\lim _{n \rightarrow \infty} \mu\left(A_{n}\right) .
$$

(iv) for every decreasing sequence $\left\{A_{n}\right\}$ of measurable sets and $\mu\left(A_{1}\right)<\infty$, we have

$$
\mu\left(\cap_{n=1}^{\infty} A_{n}\right)=\lim _{n \rightarrow \infty} \mu\left(A_{n}\right) .
$$

In many papers, a fuzzy measure is satisfying the conditions (i) and (ii). In this paper, we assume that a fuzzy

Manuscript received Jul. 14, 2010; revised Aug. 17, 2010; accepted Aug. 23, 2010 measure is satisfying the four conditions (i)-(iv).

Definition 1.1 ([2-6]) (1) The Choquet integral of a measurable function $f$ with respect to a fuzzy measure $\mu$ on $A \in \Omega$ is defined by

$$
\text { (C) } \int_{A} f d \mu=\int_{0}^{\infty} \mu(\{x \mid f(x)>r\} \cap A) d r
$$

where the integral on the right-hand side is an ordinary one.

(2) A measurable function $f$ is said to be integrable if the Choquet integral of $f$ can be defined and its value is finite.

$$
\text { Instead of }(C) \int_{X} f d \mu \text {, we will write }(C) \int f d \mu \text {. }
$$

Throughout this paper, $\mathbb{R}^{+}$will denote the interval $[0, \infty)$.

Definition 1.2 ([2-6]) A set $N \in \Omega$ is called a null set with respect to $\mu$ if $\mu(A \cup N)=\mu(A)$, for all $A \in \Omega$.

We note that $[P(x) \mu$-a.e on $A]$ means there exists a null set $N$ such that $P(x)$ is true for all $x \in A-N$, where $P(x)$ is a proposition concerning the point of $A$.

Definition 1.3 ([2-8]) Let $f, g$ be measurable nonnegative functions. We say that $f$ is comonotonic to $g$, in symbol $f \sim g$ if and only if $f(x)<f\left(x^{\prime}\right) \Rightarrow g(x) \leq g\left(x^{\prime}\right)$ for all $x, x^{\prime} \in X$.

Theorem 1.4 ([2-8]) Let $f, g, h$ be measurable functions. Then we have

(1) $f \sim f$.

(2) If $f \sim g$, then $g \sim f$.

(3) For all $a \in R^{+}$, we have $f \sim a$. 
(4) If $f \sim g$ and $f \sim h$, then $f \sim g+h$.

Theorem 1.5 ([2-8]) Let $f, g, h$ be measurable functions.

(1) If $f \leq g$, then $(C) \int f d \mu \leq(C) \int g d \mu$.

(2) If $A \subset B$ and $A, B \in \Omega$, then

$$
\text { (C) } \int_{A} f d \mu \leq(C) \int_{B} f d \mu \text {. }
$$

(3) If $f \sim g$ and $a, b \in R^{+}$, then

(C) $\int(a f+b g) d \mu=a(C) \int f d \mu+b(C) \int g d \mu$.

(4) If $(f \vee g)(x)=\max \{f(x), g(x)\}$ and

$(f \wedge g)(x)=\min \{f(x), g(x)\}$ for all $x \in X$, then

(C) $\int(f \vee g) d \mu \geq(C) \int f d \mu \vee(C) \int g d \mu$ and

(C) $\int(f \wedge g) d \mu \leq(C) \int f d \mu \wedge(C) \int g d \mu$.

Throughout this paper, $I\left(\mathbb{R}^{+}\right)$is the class of all intervals in $\mathbb{R}^{+}$, that is,

$$
I\left(\mathbb{R}^{+}\right)=\left\{\left[a^{-}, a^{+}\right] \mid a^{-}, a^{+} \in \mathbb{R}^{+} \text {and } a^{-} \leq a^{+}\right\} .
$$

For any $a \in \mathbb{R}^{+}$, we define $a=[a, a]$. Obviously, $a \in I\left(\mathbb{R}^{+}\right)$.

Definition 1.6 ([5,6]) If $\bar{a}, \bar{b} \in I\left(\mathbb{R}^{+}\right)$and $k \in \mathbb{R}^{+}$, then we define

(1) $\bar{a}+\bar{b}=\left[a^{-}+b^{-}, a^{+}+b^{+}\right]$,

(2) $k \bar{a}=\left[k a^{-}, k a^{+}\right]$,

(3) $\bar{a} \bar{b}=\left[a^{-} b^{-}, a^{+} b^{+}\right]$,

(4) $\bar{a} \wedge \bar{b}=\left[a^{-} \wedge b^{-}, a^{+} \wedge b^{+}\right]$,

(5) $\bar{a} \vee \bar{b}=\left[a^{-} \vee b^{-}, a^{+} \vee b^{+}\right]$,

(6) $\bar{a} \leq \bar{b}$ if and only if

$$
a^{-} \leq b^{-} \text {and } a^{+} \leq b^{+},
$$

(7) $\bar{a}<\bar{b}$ if and only if $\bar{a} \leq \bar{b}$ and $\bar{a} \neq \bar{b}$,

(8) $\bar{a} \subset \bar{b}$ if and only if

$$
b^{-} \leq a^{-} \text {and } a^{+} \leq b^{+} .
$$

Theorem 1.7 ([5,6]) Let $\bar{a}, \bar{b} \in I\left(\mathbb{R}^{+}\right)$. Then the followings hold.

(1) idempotent law: $\bar{a} \wedge \bar{a}=\bar{a}, \bar{a} \vee \bar{a}=\bar{a}$,

(2) commutative law:

$$
\bar{a} \wedge \bar{b}=\bar{b} \wedge \bar{a}, \bar{a} \vee \bar{b}=\bar{b} \vee \bar{a},
$$

(3) associative law:

$$
\begin{aligned}
& (\bar{a} \wedge \bar{b}) \wedge \bar{c}=\bar{a} \wedge(\bar{b} \wedge \bar{c}), \\
& (\bar{a} \vee \bar{b}) \vee \bar{c}=\bar{a} \vee(\bar{b} \vee \bar{c}),
\end{aligned}
$$

(4) absorption law:

$$
\bar{a} \wedge(\bar{a} \vee \bar{b})=\bar{a} \vee(\bar{a} \wedge \bar{b})=\bar{a},
$$

(5) distributive law:

$$
\begin{aligned}
& \bar{a} \wedge(\bar{b} \vee \bar{c})=(\bar{a} \wedge \bar{b}) \vee(\bar{a} \wedge \bar{c}), \\
& \bar{a} \vee(\bar{b} \wedge \bar{c})=(\bar{a} \vee \bar{b}) \wedge(\bar{a} \vee \bar{c}) .
\end{aligned}
$$

Definition $\quad \mathbf{1 . 8}([5,6]) \quad$ A $\quad$ set function $d_{H}: I\left(\mathbb{R}^{+}\right) \times I\left(\mathbb{R}^{+}\right) \rightarrow[0, \infty]$ is called the Hausdorff metric if

$$
\begin{array}{r}
d_{H}(A, B)=\max \left\{\sup _{x \in A} \inf _{y \in B}|x-y|,\right. \\
\left.\sup _{y \in B} \inf _{x \in A}|x-y|\right\},
\end{array}
$$

for all $A, B \in I\left(\mathbb{R}^{+}\right)$.

Theorem 1.9 ([5,6]) If $d_{H}: I\left(\mathbb{R}^{+}\right) \times I\left(\mathbb{R}^{+}\right) \rightarrow[0, \infty]$ is the Hausdorff metric, then we have for $\bar{a}=\left[a^{-}, a^{+}\right]$, $\bar{b}=\left[b^{-}, b^{+}\right] \in I\left(\mathbb{R}^{+}\right)$,

$$
d_{H}(\bar{a}, \bar{b})=\max \left\{\left|a^{-}-b^{-}\right|,\left|a^{+}-b^{+}\right|\right\} .
$$

For a sequence of intervals $\left\{\overline{a_{n}}\right\} \subset I\left(\mathbb{R}^{+}\right)$and $\bar{a} \in I\left(\mathbb{R}^{+}\right)$, we say that $\left\{\overline{a_{n}}\right\}$ converges to $\bar{a}$, in symbol, $d_{H}-\lim _{n \rightarrow \infty} \overline{a_{n}}=\bar{a}$ if $d_{H}\left(\bar{a}_{n}, \bar{a}\right) \rightarrow 0(n \rightarrow \infty)$.

Obviously, we obtain $d_{H}-\lim _{n \rightarrow \infty} \bar{a}_{n}=\bar{a}$ if and only if $a_{n}^{-} \rightarrow a^{-}$and $a_{n}^{+} \rightarrow a^{+}(n \rightarrow \infty)$.

A fuzzy number $\tilde{u}$ on $\mathbb{R}^{+}$is a fuzzy set satisfying the following conditions (see[6,9,11]);

(i)(normality) $\tilde{u}(x)=1$ for some $x \in \mathbb{R}^{+}$,

(ii)(fuzzy convexity) for every $\lambda \in(0,1]$,

$$
\widetilde{u_{\lambda}}=\left\{x \in \mathbb{R}^{+} \mid \tilde{u}(x) \geq \lambda\right\} \in I\left(\mathbb{R}^{+}\right) .
$$

Let $F N\left(\mathbb{R}^{+}\right)$denote the set of fuzzy numbers, we define basic arithmetic operations on $F N\left(\mathbb{R}^{+}\right)$(see $[6,9,11]$ ); for each pair $\tilde{u}, \tilde{v} \in F N\left(\mathbb{R}^{+}\right)$and $k \in \mathbb{R}^{+}$,

$$
(\tilde{u}+\tilde{v})_{\lambda}=\tilde{u_{\lambda}}+\widetilde{v_{\lambda}},(k \tilde{u})_{\lambda}=k \widetilde{u_{\lambda}},
$$

$\tilde{u} \leq \tilde{v}$ if and only if $\widetilde{u_{\lambda}} \leq \widetilde{v_{\lambda}}$ for all $\lambda \in(0,1]$,

$\tilde{u}<\tilde{v}$ if and only if $\tilde{u} \leq \tilde{v}$ and $\tilde{u} \neq \tilde{v}$,

In section 2, we introduce fuzzy complex numbers and discuss their basic arithmetic properties of them. And also we consider an interval-valued fuzzy measure. In section 3, we consider fuzzy valued functions and fuzzy complex valued fuzzy measures. And also we define Choquet integrals with respect to a fuzzy complex valued fuzzy measure of fuzzy complex valued functions. 


\section{Fuzzy Complex Fuzzy Measures.}

Definition 2.1 ([11]) Let $\tilde{a}, \tilde{b} \in F N\left(\mathbb{R}^{+}\right)$. We define a ordered fuzzy numbers $(\tilde{a}, \tilde{b})$ as follows:

$$
\begin{gathered}
(\tilde{a}, \tilde{b}): \mathbb{C}^{+} \rightarrow[0,1], \\
z=x+y i \mapsto(\tilde{a}, \tilde{b})(z)=\tilde{a}(x) \wedge \tilde{b}(y) .
\end{gathered}
$$

Then the mapping $(\tilde{a}, \tilde{b})$ determines a fuzzy complex number, where $\tilde{a}$ and $\tilde{b}$ is called a real part and an imaginary part of $(\tilde{a}, \tilde{b})$, respectively. Let $C=(\tilde{a}, \tilde{b})$, then $\tilde{a}=\operatorname{Re} C$, $\tilde{b}=\operatorname{Im} C$.

Let $\mathbb{C}^{+}=\left\{x+i y \mid x, y \in \mathbb{R}^{+}\right\}$and $F C N\left(\mathbb{C}^{+}\right)$be the set of fuzzy complex numbers on $\mathbb{C}^{+}$, writing

$$
\tilde{c}=\tilde{a}+\tilde{b} i=(\tilde{a}, \tilde{b}), \tilde{a}, \tilde{b} \in F N\left(\mathbb{R}^{+}\right) .
$$

We note that if $c=a+b i$ is a complex number, then it's membership function is

$$
c(z)=\left\{\begin{array}{cc}
1 & x=a, y=b \\
0 & \text { otherwise }
\end{array}\right.
$$

whenever $z=(x, y) \in \mathbb{C}^{+}$. If $C_{1}, C_{2} \in F C N\left(\mathbb{C}^{+}\right)$and we define

$$
C_{1} * C_{2}=\left(\operatorname{Re} C_{1} * \operatorname{Re} C_{2}, \operatorname{Im} C_{1} * \operatorname{Im} C_{2}\right)
$$

for operation $* \in\{+,-, \bullet, \wedge, \vee\}$, then clearly we have $C_{1} * C_{2}$ belongs to $F C N\left(\mathbb{C}^{+}\right)$. Now we introduce some order relations and equality relation on $F C N\left(\mathbb{C}^{+}\right)$.

Definition 2.2 ([11]) Let $C_{1}, C_{2} \in F C N\left(\mathbb{C}^{+}\right)$.

(1) $C_{1} \leq C_{2}$ if and only if

$$
\operatorname{Re} C_{1} \leq \operatorname{Re} C_{2}, \operatorname{Im} C_{1} \leq \operatorname{Im} C_{2} .
$$

(2) $C_{1}<C_{2}$ if and only if $C_{1} \leq C_{2}$ and $\operatorname{Re} C_{1}<$ $\operatorname{Re} C_{2}$ or $\operatorname{Im} C_{1}<\operatorname{Im} C_{2}$.

(3) $C_{1}=C_{2}$ if and only if

$$
C_{1} \leq C_{2}, C_{2} \leq C_{1} .
$$

We will define the new metric $D$ on $F C N\left(\mathbb{C}^{+}\right)$as follows.

Definition 2.3 (1) If $C \in F C N\left(\mathbb{C}^{+}\right)$, then $C_{\lambda}$ is closed rectangle region on $\mathbb{C}^{+}$, for all $\lambda \in(0,1]$, defined as $C_{\lambda}=\left\{z \in \mathbb{C}^{+} \mid C(z) \geq \lambda\right\} . \quad$ Obviously, if $\tilde{a}, \tilde{b} \in F N\left(\mathbb{R}^{+}\right)$, then $(\tilde{a}, \tilde{b})_{\lambda}=\left(\tilde{a}_{\lambda}, \tilde{b}_{\lambda}\right)$ for all $\lambda \in(0,1]$.

(2) A mapping

$$
D: F C N\left(\mathbb{C}^{+}\right) \times F C N\left(\mathbb{C}^{+}\right) \rightarrow[0, \infty]
$$

is defined by

$$
\begin{aligned}
& D\left(C_{1}, C_{2}\right) \\
& =\max \left\{\Delta\left(\operatorname{Re} C_{1}, \operatorname{Re} C_{2}\right), \Delta\left(\operatorname{Im} C_{1}, \operatorname{Im} C_{2}\right)\right\}
\end{aligned}
$$

where

$$
\begin{aligned}
& \Delta\left(\operatorname{Re} C_{1}, \operatorname{Re} C_{2}\right) \\
& =\vee_{\lambda \in(0,1]} d_{H}\left(\left(\operatorname{Re} C_{1}\right)_{\lambda},\left(\operatorname{Re} C_{2}\right)_{\lambda}\right)
\end{aligned}
$$

and

$$
\begin{aligned}
& \Delta\left(\operatorname{Im} C_{1}, \operatorname{Im} C_{2}\right) \\
& =\vee_{\lambda \in(0,1]} d_{H}\left(\left(\operatorname{Im} C_{1}\right)_{\lambda},\left(\operatorname{Im} C_{2}\right)_{\lambda}\right) .
\end{aligned}
$$

It is clearly to see that $\left(F C N\left(\mathbb{C}^{+}\right), D\right)$ is a metric space. By using this metric, we define the concept of convergence of a sequence in the metric space $\left(F C N\left(\mathbb{C}^{+}\right), D\right)$.

Definition 2.4 Let $\left\{C_{n}\right\} \subset F C N\left(\mathbb{C}^{+}\right)$be a sequence of fuzzy complex valued numbers and $C \in F C N\left(\mathbb{C}^{+}\right)$. The sequence $\left\{C_{n}\right\}$ converges to $C$, in symbol, $D-\lim _{n \rightarrow \infty} C_{n}=C$ if

$$
\lim _{n \rightarrow \infty} D\left(C_{n}, C\right)=0 .
$$

We also consider an interval-valued fuzzy measure as follows.

Definition 2.5 ([11]) Let $(X, \Omega)$ be a measurable space, a mapping

$\bar{\mu}: \Omega \rightarrow I\left(\mathbb{R}^{+}\right)$is called an interval-valued fuzzy measure if it is satisfying

(i) $\bar{\mu}(\varnothing)=[0,0]$,

(ii) $\bar{\mu}(A) \leq \bar{\mu}(B)$, whenever

$A, B \in \Omega, A \subset B$.

(iii) If $\left(A_{n}\right) \subset \Omega$ and $A_{n} \nearrow A$ or $A_{n} \searrow A$ implies $\bar{\mu}\left(A_{n}\right) \rightarrow \bar{\mu}(A)$.

We note that for any $A \in \Omega$, denote $\bar{\mu}(A)=\left[\mu^{-}(A), \mu^{+}(A)\right]$ or simply write as $\bar{\mu}=\left[\mu^{-}, \mu^{+}\right]$.

Proposition 2.6 ([11]) A mapping $\bar{\mu}: \Omega \rightarrow I\left(\mathbb{R}^{+}\right)$is an interval-valued fuzzy measure if and only if $\mu^{-}$and $\mu^{+}$are fuzzy measure under Sugeno's sense.

\section{Choquet Integrals of Fuzzy Complex Valued Functions}

In this section, we define a fuzzy complex valued fuzzy measure and Choquet integral with respect to a fuzzy complex valued fuzzy measure of fuzzy complex valued functions. Let $\mathbb{C}^{+}=\left\{x+y i \mid x, y \in \mathbb{R}^{+}\right\}$and $\left(\mathbb{C}^{+}, \Omega\right)$ be a measurable space. We consider a fuzzy complex valued function as follows.

Definition 3.1 ([11]) If a mapping $\tilde{f}: \mathbb{C}^{+} \rightarrow F C N\left(\mathbb{C}^{+}\right)$is defined by 


$$
\begin{aligned}
z=x+y i \rightarrow \tilde{f}(z) & =(\operatorname{Re} \tilde{f}, \operatorname{Im} \tilde{f})(z) \\
& \equiv \operatorname{Re} \tilde{f}(x) \wedge \operatorname{Im} \tilde{f}(y),
\end{aligned}
$$

then $\tilde{f}$ is called a fuzzy complex valued function on $\mathbb{C}^{+}$.

We note that $\operatorname{Re} \tilde{f}(x) \in F N\left(\mathbb{R}^{+}\right), \operatorname{Im} \tilde{f}(y) \in F\left(\mathbb{R}^{+}\right)$. For any $\lambda \in(0,1]$, let

$$
\begin{gathered}
\widetilde{f}_{\lambda}(z)=(\tilde{f}(z))_{\lambda}=\left(\operatorname{Re} \widetilde{f_{\lambda}}(z), \operatorname{Im} \widetilde{f}_{\lambda}(z)\right), \\
(\operatorname{Re} \tilde{f})_{\lambda}=\operatorname{Re} \widetilde{f_{\lambda}}=\left(\operatorname{Re} \widetilde{f}_{\lambda}{ }^{-}, \operatorname{Re}{\widetilde{f_{\lambda}}}^{+}\right), \text {and } \\
(\operatorname{Im} \tilde{f})_{\lambda}=\operatorname{Im} \widetilde{f_{\lambda}}=\left(\operatorname{Im}{\widetilde{f_{\lambda}}}^{-}, \operatorname{Im} \widetilde{f}_{\lambda}{ }^{+}\right) .
\end{gathered}
$$

Theorem 3.2 ([11]) Let $\tilde{f}_{1}, \widetilde{f}_{2}$ be fuzzy complex valued measurable functions on $\left(\mathbb{C}^{+}, \Omega\right)$, then $\tilde{f}_{1} \pm \widetilde{f}_{2}$ and $\tilde{f}_{1} \cdot \tilde{f}_{2}$ are fuzzy complex valued measurable functions.

Definition 3.3 ([11]) Let $\left(\mathbb{C}^{+}, \Omega\right)$ be a measurable space, a mapping $\tilde{\mu}: \Omega \rightarrow F C N\left(\mathbb{C}^{+}\right)$is called a fuzzy complex valued fuzzy measure, if the following conditions are satisfied:

(1) $\tilde{\mu}(\varnothing)=[\tilde{0}, \tilde{0}]$, where $\tilde{0} \in F C N\left(\mathbb{C}^{+}\right)$,

(2) $\tilde{\mu}(A) \leq \tilde{\mu}(B)$,

whenever $A, B \in \Omega, A \subset B$, and

(3) if $\left(A_{n}\right) \subset \Omega$ and $A_{n} \nearrow A$ or $A_{n} \searrow A$, then $\tilde{\mu}\left(A_{n}\right) \rightarrow \tilde{\mu}(A) \quad(n \rightarrow \infty)$ in meaning of the metric $D$, in symbol,

$$
D-\lim _{n \rightarrow \infty} \tilde{\mu}_{n}=\tilde{\mu}
$$

We note that $\left(\mathbb{C}^{+}, \Omega, \tilde{\mu}\right)$ is called a fuzzy complex valued fuzzy measure space and denote that $\tilde{\mu}(A)=\left(\tilde{\mu}_{R}(A), \tilde{\mu}_{I}(A)\right)$ or simply write as $\tilde{\mu}=\left(\tilde{\mu}_{R}, \tilde{\mu}_{I}\right)$ for any $A \in \Omega$. Now, we will define the Choquet integral with respect to a fuzzy complex fuzzy measure of a fuzzy complex valued function as follows. The idea of the following definition is similar to the idea of the generalized Lebesgue integral in Wang and $\mathrm{Li}$ [11].

Definition 3.4 Let $\tilde{\mu}=\left(\tilde{\mu}_{R}, \tilde{\mu}_{I}\right)$ be a fuzzy complex valued fuzzy measure and $\tilde{f}=(\operatorname{Re} \tilde{f}, \operatorname{Im} \tilde{f})$ a fuzzy complex valued measurable function.

(1) For any $A \in \Omega$, the Choquet integral with respect to $\tilde{\mu}$ of $\tilde{f}$ is defined by

$$
\begin{gathered}
\left((C) \int_{A} \tilde{f} d \tilde{\mu}\right)_{\lambda} \equiv\left((C) \int_{A} \operatorname{Re} \tilde{f}_{\lambda} d\left(\tilde{\mu}_{R}\right)_{\lambda},\right. \\
\left.(C) \int_{A} \operatorname{Im} \tilde{f}_{\lambda} d\left(\tilde{\mu}_{I}\right)_{\lambda}\right)
\end{gathered}
$$

for all $\lambda \in(0,1]$, where

(C) $\int_{A} \operatorname{Re} \tilde{f}_{\lambda} d\left(\tilde{\mu}_{R}\right)_{\lambda}=$

$$
\left[(C) \int_{A}(\operatorname{Re} \tilde{f})_{\lambda}^{-} d\left(\tilde{\mu}_{R}\right)_{\lambda}^{-},(C) \int_{A}(\operatorname{Re} \tilde{f})_{\lambda}^{+} d\left(\tilde{\mu}_{R}\right)_{\lambda}^{+}\right]
$$

and

(C) $\int_{A} \operatorname{Im} \tilde{f}_{\lambda} d\left(\tilde{\mu}_{I}\right)_{\lambda}=$

$$
\left[(C) \int_{A}(\operatorname{Im} \tilde{f})_{\lambda}^{-} d\left(\tilde{\mu}_{I}\right)_{\lambda}^{-},(C) \int_{A}(\operatorname{Im} \tilde{f})_{\lambda}^{+} d\left(\tilde{\mu}_{I}\right)_{\lambda}^{+}\right] .
$$

(2) If there exists $\tilde{u} \in F C N\left(\mathbb{C}^{+}\right)$such that $(\tilde{u})_{\lambda}=\left((C) \int_{A} \tilde{f} d \tilde{\mu}\right)_{\lambda}$ for all $\lambda \in(0,1]$, then $\tilde{f}$ is said to be Choquet integrable on $A$.

(3) $\tilde{f}$ is said to be Choquet integrably bounded if $R e \tilde{f}$ and $\operatorname{Im} \tilde{f}$ are Choquet integrably bounded.

$$
\text { Instead of }(C) \int_{X} \tilde{f} d \tilde{\mu} \text {, we will write }(C) \int \tilde{f} d \tilde{\mu} \text {. }
$$

Remark 3.5 Re $\tilde{f}$ and $\operatorname{Im} \tilde{f}$ are Choquet integrably bounded if and only if for all $\lambda \in(0,1]$, interval-valued measurable functions $(\operatorname{Re} \tilde{f})_{\lambda}$ and $(\operatorname{Im} \tilde{f})_{\lambda}$ are Choquet integrably bounded (see $[5,6])$. And we also see that

$$
\begin{aligned}
& (C) \int_{A}(\operatorname{Re} \tilde{f})_{\lambda}^{-} d\left(\tilde{\mu}_{R}\right)_{\lambda}^{-},(C) \int_{A}(\operatorname{Re} \tilde{f})_{\lambda}^{+} d\left(\tilde{\mu}_{R}\right)_{\lambda}^{+}, \\
& (C) \int_{A}(\operatorname{Im} \tilde{f})_{\lambda}^{-} d\left(\tilde{\mu}_{I}\right)_{\lambda}^{-} \text {and }(C) \int_{A}(\operatorname{Im} \tilde{f})_{\lambda}^{+} d\left(\tilde{\mu}_{I}\right)_{\lambda}^{+}
\end{aligned}
$$

are finite, that is, they are well-defined (see [2-4]).

Lemma 3.6 ([6,9]) Let $\left\{\left[a_{\lambda}, b_{\lambda}\right] \mid \lambda \in(0,1]\right\}$ be given a family of nonempty intervals in $I\left(\mathbb{R}^{+}\right)$. If (i) for all $0<\lambda_{1} \leq \lambda_{2}, \quad\left[a_{\lambda_{1}}, b_{\lambda_{1}}\right] \supset\left[a_{\lambda_{2}}, b_{\lambda_{2}}\right]$ and (ii) for any nonincreasing sequence $\left\{\lambda_{k}\right\}$ in $(0,1]$ converging to $\lambda$, $\left[a_{\lambda}, b_{\lambda}\right]=\bigcap_{k=1}^{\infty}\left[a_{\lambda_{k}}, b_{\lambda_{k}}\right]$. Then there exists a unique fuzzy number $\tilde{u} \in F N\left(\mathbb{R}^{+}\right)$such that the family $\left[a_{\lambda}, b_{\lambda}\right]$ represents the $\lambda$-level sets of a fuzzy number $\tilde{u} \in F N\left(\mathbb{R}^{+}\right)$.

Conversely, if $\left[a_{\lambda}, b_{\lambda}\right]$ are the $\lambda$-level set of a fuzzy number $\tilde{u} \in F N\left(\mathbb{R}^{+}\right)$, then the conditions (i) and (ii) are satisfied.

By using the definition of a fuzzy complex valued fuzzy measure with condition (iii), we easily obtain the following lemma.

Lemma 3.7 Let $\left\{\lambda_{k}\right\}$ be a nonincreasing sequence in $(0,1]$ converging to $\lambda$. If we put

$$
\begin{gathered}
g_{k, m}^{R}(\alpha)=\left(\tilde{\mu}_{R}\right)_{\lambda_{k}}^{-}\left(\left\{x \mid(R e \tilde{f})_{\lambda_{m}}^{-}(x)>\alpha\right\}\right), \\
h_{k, m}^{R}(\alpha)=\left(\tilde{\mu}_{R}\right)_{\lambda_{k}}^{+}\left(\left\{x \mid(R e \tilde{f})_{\lambda_{m}}^{+}(x)>\alpha\right\}\right), \text { and }
\end{gathered}
$$




$$
\begin{gathered}
g_{k, m}^{I}(\alpha)=\left(\tilde{\mu}_{I}\right)_{\lambda_{k}}^{-}\left(\left\{x \mid(\operatorname{Im} \tilde{f})_{\lambda_{m}}^{-}(x)>\alpha\right\}\right), \\
h_{k, m}^{I}(\alpha)=\left(\tilde{\mu}_{I}\right)_{\lambda_{k}}^{+}\left(\left\{x \mid(\operatorname{Im} \tilde{f})_{\lambda_{m}}^{+}(x)>\alpha\right\}\right), \text { and } \\
g_{\lambda}^{R}(\alpha)=\left(\tilde{\mu}_{R}\right)_{\lambda}^{-}\left(\left\{x \mid(\operatorname{Re} \tilde{f})_{\lambda}^{-}(x)>\alpha\right\}\right), \\
h_{\lambda}^{R}(\alpha)=\left(\tilde{\mu}_{I}\right)_{\lambda}^{+}\left(\left\{x \mid(\operatorname{Re} \tilde{f})_{\lambda}^{+}(x)>\alpha\right\}\right), \text { and } \\
g_{\lambda}^{I}(\alpha)=\left(\tilde{\mu}_{T}\right)_{\lambda}^{-}\left(\left\{x \mid(\operatorname{Im} \tilde{f})_{\lambda}^{-}(x)>\alpha\right\}\right), \\
h_{\lambda}^{I}(\alpha)=\left(\tilde{\mu}_{I}\right)_{\lambda}^{+}\left(\left\{x \mid(\operatorname{Im} \tilde{f})_{\lambda}^{+}(x)>\alpha\right\}\right),
\end{gathered}
$$

for all $\alpha \in \mathbb{R}^{+}$and $k, m \in \mathbb{N}$, then we have $g_{k, m}^{R}(\alpha) \searrow g_{\lambda}^{R}(\alpha), h_{k, m}^{R}(\alpha) \searrow h_{\lambda}^{R}(\alpha), g_{k, m}^{I}(\alpha) \searrow g_{\lambda}^{I}(\alpha)$, and $h_{k, m}^{I}(\alpha) \searrow h_{\lambda}^{I}(\alpha)$.

Remark 3.8 (1) For all $0<\lambda_{1} \leq \lambda_{2}$, we obtain

$$
\begin{aligned}
& \text { (C) } \int_{A}(\operatorname{Re} \tilde{f})_{\lambda_{1}}^{-} d\left(\tilde{\mu}_{R}\right)_{\lambda_{1}}^{-} \geq(C) \int_{A}(\operatorname{Re} \tilde{f})_{\lambda_{2}}^{-} d\left(\tilde{\mu}_{R}\right)_{\lambda_{2}}^{-} \text {, } \\
& \text { (C) } \int_{A}(\operatorname{Re} \tilde{f})_{\lambda_{1}}^{+} d\left(\tilde{\mu}_{R}\right)_{\lambda_{1}}^{+} \geq(C) \int_{A}(\operatorname{Re} \tilde{f})_{\lambda_{2}}^{+} d\left(\tilde{\mu}_{R}\right)_{\lambda_{2}}^{+} \text {, } \\
& \text { (C) } \int_{A}(\operatorname{Im} \tilde{f})_{\lambda_{1}}^{-} d\left(\tilde{\mu}_{I}\right)_{\lambda_{1}}^{-} \geq(C) \int_{A}(\operatorname{Im} \tilde{f})_{\lambda_{2}}^{-} d\left(\tilde{\mu}_{I}\right)_{\lambda_{2}}^{-} \text {, } \\
& \text { and } \\
& \text { (C) } \int_{A}(\operatorname{Im} \tilde{f})_{\lambda_{1}}^{+} d\left(\tilde{\mu}_{I}\right)_{\lambda_{1}}^{+} \geq(C) \int_{A}(\operatorname{Im} \tilde{f})_{\lambda_{2}}^{+} d\left(\tilde{\mu}_{I}\right)_{\lambda_{2}}^{+} \text {. }
\end{aligned}
$$

(2) If we take $k=m$, then we obtain the followings: For any nonincreasing sequence $\left\{\lambda_{k}\right\}$ in $(0,1]$ conversing to $\lambda$,

$$
\begin{aligned}
& (C) \int_{A}(\operatorname{Re} \tilde{f})_{\lambda}^{-} d\left(\tilde{\mu}_{R}\right)_{\lambda}^{-} \\
= & \bigcap_{k=1}^{\infty}\left((C) \int_{A}(\operatorname{Re} \tilde{f})_{\lambda_{k}}^{-} d\left(\tilde{\mu}_{R}\right)_{\lambda_{k}}^{-},\right. \\
& (C) \int_{A}(\operatorname{Re} \tilde{f})_{\lambda}^{+} d\left(\tilde{\mu}_{R}\right)_{\lambda}^{+} \\
= & \left.\bigcap_{k=1}^{\infty}(C) \int_{A}(\operatorname{Re} \tilde{f})_{\lambda_{k}}^{+} d\left(\tilde{\mu}_{R}\right)_{\lambda_{k}}^{+}\right), \\
(C) \int_{A}(\operatorname{Im} \tilde{f})_{\lambda}^{-} d(\tilde{\mu})_{I}^{-} & \bigcap_{k=1}^{\infty}\left((C) \int_{A}(\operatorname{Im} \tilde{f})_{\lambda_{k}}^{-} d\left(\tilde{\mu}_{I}\right)_{\lambda_{k}}^{-}\right), \\
& (C) \int_{A}(\operatorname{Im} \tilde{f})_{\lambda}^{+} d\left(\tilde{\mu}_{I}\right)_{\lambda}^{+} \\
= & \bigcap_{k=1}^{\infty}\left((C) \int_{A}(\operatorname{Im} \tilde{f})_{\lambda_{k}}^{+} d\left(\tilde{\mu}_{I}\right)_{\lambda_{k}}^{+}\right) .
\end{aligned}
$$

From Definition 3.4, Remark 3.5, Lemma 3.7 and Remark 3.8, we obtain the following theorem.

Theorem 3.9 Let $\left(\mathbb{C}^{+}, \Omega, \tilde{\mu}\right)$ be fuzzy complex valued fuz- zy measure space. If $\tilde{f}$ is a fuzzy complex valued integrably bounded function, then we have

(i) for all $0<\lambda_{1} \leq \lambda_{2}$,

$$
\left((C) \int_{A} \tilde{f} d \tilde{\mu}\right)_{\lambda_{1}} \supset\left((C) \int_{A} \tilde{f} d \tilde{\mu}\right)_{\lambda_{2}},
$$

(ii) for any nonincreasing sequence $\left\{\lambda_{k}\right\}$ in $(0,1]$ conversing to $\lambda$,

$$
\left((C) \int_{A} \tilde{f} d \tilde{\mu}\right)_{\lambda}=\bigcap_{k=1}^{\infty}\left((C) \int_{A} \tilde{f} d \tilde{\mu}\right)_{\lambda_{k}} .
$$

Remark 3.10 From Theorem 3.9 and Lemma 3.6, there exists a fuzzy complex number $\tilde{u} \in F C N\left(\mathbb{C}^{+}\right)$such that

$$
(\tilde{u})_{\lambda}=\left((C) \int_{A} \tilde{f} d \tilde{\mu}\right)_{\lambda}
$$

for all $\lambda \in(0,1]$. That is, if a fuzzy complex valued function $\tilde{f}$ is integrably bounded, then it is Choquet integrable.

\section{References}

[1] J.J. Buckley, "Fuzzy complex numbers", Fuzzy Set and Systems vol.33, pp.333-345, 1989.

[2] L. C. Jang, T. Kim, J. Jeon, "On set-valued Choquet integrals and convergence theorems", Advan. Stud. Contemp. Math. vol.6, pp.63-76, 2003.

[3] L. C. Jang, "A study on applications of Choquet integral on interval -valued fuzzy sets", Proceedings of the Jangjeon Mathematical Society vol.10, pp.161-172, 2007.

[4] L.C. Jang, "A note on the monotone interval-valued set function defined by the interval-valued Choquet integral", Commun. Korean Math. Soc. vol.22, no.2, pp.227-234, 2007.

[5] L.C. Jang, "Structural characterizations of monotone interval-valued set functions defined by the interval-valued Choquet integral", Fuzzy Logic and Intelligent vol.18, no.3, pp.311-315, 2008.

[6] L.C. Jang, T. Kim, J.D. Jeon, and W.J. Kim, "On Choquet integrals of measurable fuzzy number-valued functions", Bull.KoreanMath.Soc. vol. 41, no. 1, pp.95-107, 2004.

[7] T. Murofush and M. Sugeno, "An interpretation of fuzzy measures and the Choquet integral as an integral with respect to a fuzzy measure", Fuzzy Sets and Systems vol. 29, pp. 201-227, 1989.

[8] T. Murofush and M. Sugeno, "A theory of fuzzy measures: representations, the Choquet integral, and null sets", J.Math. Anal. Appl. vol.159, pp. 531-549, 1991.

[9] M.L. Puri and D.A. Ralescu, "Fuzzy random variable", J.Math. Anal. Appl. vol.114, pp. 409-422, 1986.

[10] M. Sugeno, Y. Narukawa and T. Murofushi, "Choquet 
integral and fuzzy measures on locally compact space", Fuzzy Sets and Systems vol.99,pp.205-211,1998.

[11] Guijin Wang and Xiaoping Li, "Generalized Lebesgue integrals of fuzzy complex valued functions", Fuzzy Sets and Systems vol.127, pp.363-370, 2002.

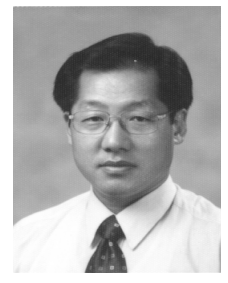

Lee-Chae Jang received his MS and Ph.D at Kyungpook University under the direction of Han-Soo Kim. Since 1987 he has been a professor at Konkuk University. His research interest is analysis, fuzzy measure and Choquet integral, information theory, and p-adic analysis.
Department of Mathematics and Computer Science, Konkuk University, Chungju, 380-701, Korea.

E-mail: leechae.jang@kku.ac.kr

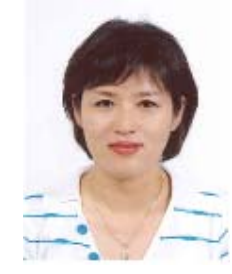

Hyun-Mee Kim received her Ph.D at Kyunghee University under the direction of Jong-Duck Jeon. Since 1995 she has been a parttime instructor at Kyunghee University and Konkuk University, etc. Her research interest is fuzzy theory and functional analysis.

E-mail: kagness@khu.ac.kr 